\title{
Dosimètre individuel électronique pour les neutrons : Saphydose-n
}

\author{
T. LAHAYE*, D. CUTARELLA*, S. MÉNARD*, A. RANNOU*, \\ T. BOLOGNESE-MILSZTAJN*
}

(Manuscrit reçu le 7 mars 2000, accepté le 17 avril 2000)

RÉSUMÉ La dosimétrie individuelle des neutrons constitue une des difficultés actuelles de la radioprotection des travailleurs. La dosimétrie dite active (opérationneIle) est réglementaire depuis mars 1999 ; les valeurs des seuils recommandées par la CIPR 60 applicables en mai 2000 rendent nécessaire la mise en place d'une nouvelle génération de dosimètres neutrons. L'Institut de protection et de sûreté nucléaire (IPSN) a mené, au cours de ces dernières années, l'étude d'un dosimètre électronique individuel pour les neutrons satisfaisant aux spécifications d'un cahier des charges défini par le groupe de travail « dosimétrie neutrons » réunissant les principaux acteurs du cycle nucléaire français. Courant 1998, I'IPSN a engagé un transfert de technologie vers la société Saphymo ; celle-ci commercialisera fin 2000 le premier dosimètre individuel électronique pour les neutrons, le Saphydose-n, répondant à la norme CEI 1323. Ce dosimètre, d'un encombrement réduit, permettra d'évaluer l'équivalent de dose individuel $\mathrm{Hp}$ (10) dans des champs mixtes neutron-gamma. Il pourra être utilisé dans toutes les installations du cycle électronucléaire, sans connaissance préalable de l'énergie moyenne du spectre des neutrons et du rapport neutron-gamma. Le dosimètre Saphydose-n pourra être raccordé aux bornes existantes des dosimètres gamma pour la lecture des informations et pour la charge des accumulateurs.

ABSTRACT Electronic personal dosemeter for neutrons: Saphydose-n.

Personal neutron dosimetry represents one of the current difficulties in the field of radiological protection of workers. Since March 1999, the regulatory requirements for « active » (i.e. operational) dosimetry have been those of ICRP Publication 60, applicable from May 2000 , necessitating the introduction of a new generation of neutron dosimeters. Over the last few years, the Institute for Nuclear Safety and Protection (IPSN) has been studying an electronic personal dosimeter for neutrons, capable of meeting the specifications laid down by a neutron dosimetry-working group including members from all the main players in the French nuclear industry. In 1998, the IPSN began transferring technology to the company Saphymo which, by the end of 2000 , will be marketing Saphydose-n, the first personal dosimeter for neutrons complying with IEC 1323. This dosimeter is of compact design and can assess the personal dose equivalent $\mathrm{Hp}(10)$ in mixed neutron and gamma radiation fields. It will be useable in any nuclear facility without prior knowledge of the average neutron spectrum or the neutron-gamma ratio. It will be possible to connect the Saphydose-n dosimeter to any of the existing gamma dosimeter terminals to read the dose data and recharge the batteries.

Institut de protection el de sûrcté nucléaire, DPHD/SDOS, BP 6, 92265 Fontenay-aux-Roses Cedex, France. 


\section{Expression des besoins en dosimétrie des neutrons}

Le groupe de travail « dosimétrie neutrons » (Rannou et al., 1997) constitué des représentants d'EDF, de la COGEMA, de l'ETCA, du CEA et de l'IPSN a exprimé en 1993 ses besoins en termes de dosimétrie individuelle opérationnelle pour les neutrons. Un cahier des charges spécifiant les caractéristiques du dosimètre individuel électronique « idéal » pour les neutrons, a été rédigé. Ce dosimètre doit en particulier répondre aux recommandations de la norme CEI 1323. Les principales caractéristiques doivent être les suivantes :

- détecter les neutrons d'énergie comprise entre $10^{-8} \mathrm{MeV}$ (énergie thermique) et $15 \mathrm{MeV}$;

- discriminer la composante neutron de la composante gamma d'un champ de rayonnements mixtes « neutron-gamma »;

- évaluer la grandeur opérationnelle $\mathrm{Hp}(10)$ neutron (équivalent de dose individuel);

- disposer d'une dynamique de mesure comprise entre $10 \mu \mathrm{Sv} \mathrm{h}^{-1}$ et $1 \mathrm{~Sv} \mathrm{~h}^{-1}$;

- afficher et signaler par une alarme sonore le dépassement d'un seuil pré-défini en équivalent de dose individuel $\mathrm{Hp}(10)$ et en débit d'équivalent de dose individuel $\mathrm{H} p$;

- fonctionner dans les conditions environnementales habituellement exigées pour la dosimétrie individuelle (température, hygrométrie, compatibilité électromagnétique, choc, etc.).

Le seul dosimètre donnant une réponse en temps réel étant le dosimètre à bulles, le groupe de travail a recommandé sa mise en place pour la gestion journalière des équivalents de doses intégrés par les agents du cycle électronucléaire. Cette dosimétrie opérationnelle a ainsi contribué à l'application du principe ALARA, en informant l'agent de l'équivalent de dose reçu à tout moment de son intervention, par lecture directe. Le retour d'expérience des utilisateurs montre toutefois que les dosimètres à bulles ne peuvent pas satisfaire à l'ensemble de leurs besoins. Ces dosimètres ne pourront pas répondre aux spécifications requises par les normes européennes.

\section{Grandeurs dosimétriques de radioprotection}

La Commission internationale de protection radiologique (CIPR) définit, dans la publication $\mathrm{n}^{\circ} 60$, les trois grandeurs de radioprotection : la dose absorbée « $\mathrm{D}$ », la dose équivalente « $\mathrm{H}_{\mathrm{T}}$ » et la dose efficace « $\mathrm{E} »$. Ces grandeurs n'étant pas directement mesurables, la grandeur recommandée est l'équivalent de dose individuel $\mathrm{Hp}(10)$, 


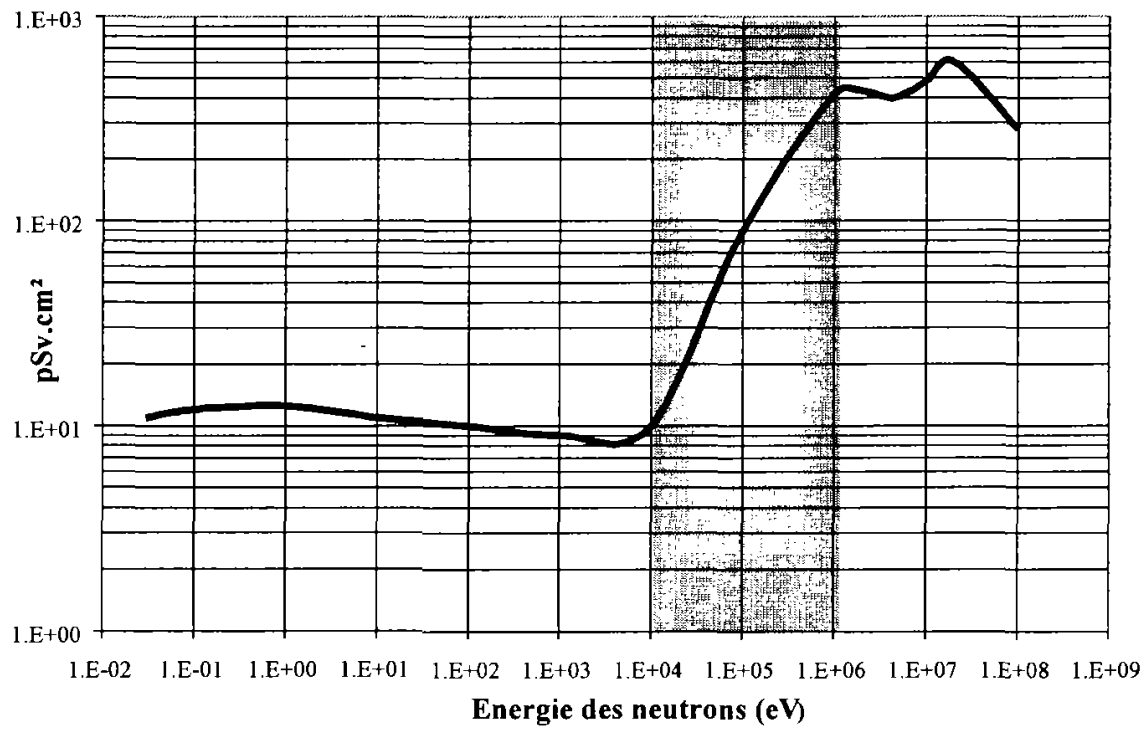

Fig. 1. - Variation du coefficient de conversion $h(E)$ en fonction de l'énergie des neutrons (incidence $=0^{\circ}$ ). Conversion coefficient $h(E)$ as a function of the energy of neutrons (incidence $=\theta^{\circ}$ ).

sous $10 \mathrm{~mm}$ de tissu biologique. Il est calculé à partir du spectre en fluence et en énergie du champ de rayonnement en appliquant un coefficient de conversion dépendant de l'énergie et de l'angle d'incidence des neutrons, « $\mathrm{h}_{(\mathrm{E})} »$, fourni par la norme ISO 8529. Ce coefficient présente de grandes variations en fonction de l'énergie des neutrons, notamment entre $10^{4}$ et $10^{6} \mathrm{eV}$ (Fig. 1).

Ce domaine d'énergie (en grisé sur la Fig. 1) est celui pour lequel l'équivalent de dose dans les installations nucléaires est le plus important (même en présence de neutrons thermiques).

La détermination du spectre en énergie d'un champ de rayonnement neutronique nécessite la mise en œuvre d'une technologie complexe dont l'encombrement est encore aujourd'hui incompatible avec les dimensions d'un dosimètre individuel. La seule solution, pour répondre aux spécifications de la norme CEI 1323, est de concevoir un dosimètre dont la réponse en termes d'équivalent de dose individuel $(\mathrm{Hp}(10))$ soit indépendante de l'énergie des neutrons incidents. Les champs mixtes rencontrés dans les installations nucléaires comprennent souvent des neutrons rétrodiffusés par l'environnement de travail (murs, sol et objets). La distribution en énergie des neutrons présente des formes variées d'une installation à l'autre, si bien que l'évaluation de l'équivalent de dose individuel par un dosimètre neutron dont la réponse dépendrait de l'énergie serait impossible. 


\section{Développement par l'IPSN d'un dosimètre opérationnel}

L'objectif du service de dosimétrie de l'IPSN a été de concevoir un dosimètre individuel neutron répondant à l'ensemble des spécifications du cahier des charges du groupe de travail «dosimétrie neutrons » et de la norme CEI 1323. La principale difficulté était d'obtenir un appareil dont la réponse en terme d'équivalent de dose soit indépendante de l'énergie des neutrons incidents. Plusieurs voies technologiques ont été explorées par l'IPSN au cours des dernières années et ont conduit au développement d'un dosimètre à base d'un détecteur semi-conducteur.

\section{Principe de détection}

Les détecteurs semi-conducteurs (silicium) déjà utilisés en dosimétrie individuelle pour les rayonnements $\mathrm{X}$ et gamma répondent de façon satisfaisante aux spécifications techniques requises par les normes. De plus, ils sont robustes et peu encombrants. La section efficace d'interaction nucléaire des neutrons avec le silicium est trop faible pour que ce composant soit directement utilisé comme détecteur neutron. La section efficace d'interaction des neutrons avec un milieu fortement hydrogéné est plus importante que celle des neutrons avec le silicium ; le service de dosimétrie de l'IPSN a développé un convertisseur «neutrons- particules chargées » à base de polyéthylène. Ce convertisseur de faible encombrement a été optimisé pour que la réponse du dosimètre soit correcte sur l'ensemble de la gamme d'énergies des neutrons (des énergies thermiques à $15 \mathrm{MeV}$ ). Il est constitué d'un film de polyéthylène implanté d'atomes de ${ }^{10} \mathrm{~B}$ et il est disposé directement au contact de la fenêtre d'entrée de la diode silicium (Fig. 2).

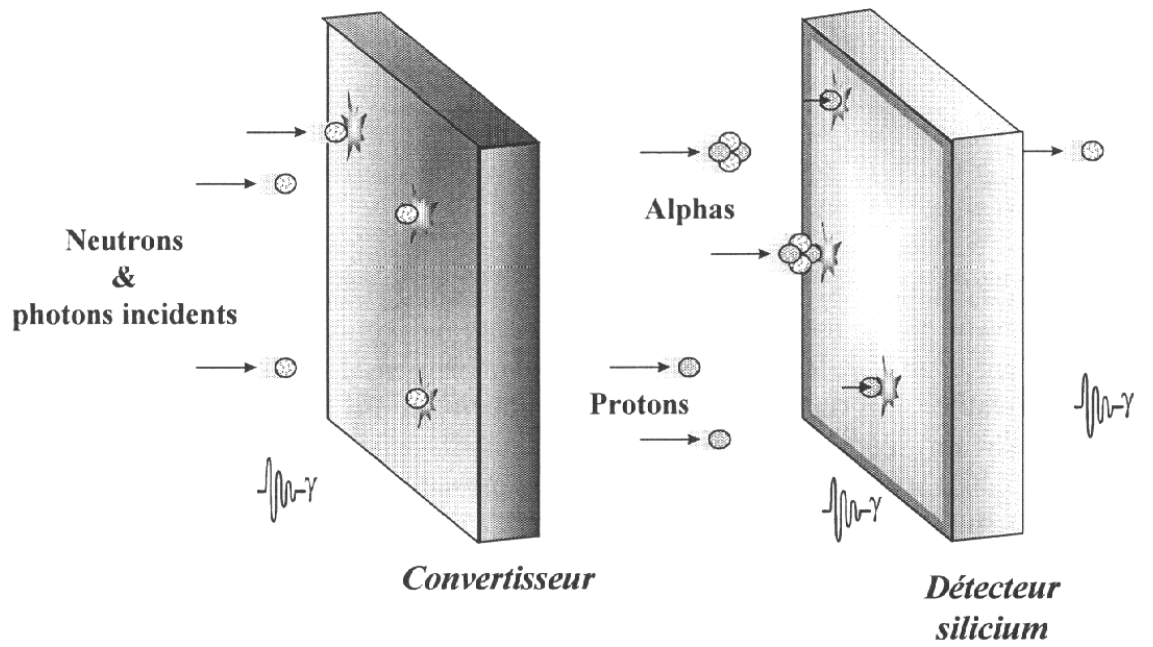

Fig. 2. - Principe de détection.

Detection principle. 
Les neutrons interagissent avec les atomes de carbone et d'hydrogène du polyéthylène ou avec les atomes de bore. Les neutrons d'énergie supérieure à $10 \mathrm{keV}$ interagissent majoritairement avec le polyéthylène par diffusion élastique. Lors de ces collisions, une partie de l'énergie des neutrons incidents est transférée aux protons des atomes du polyéthylène. Ce transfert d'énergie a pour conséquence la mise en mouvement des protons. Les neutrons d'énergie inférieure à $10 \mathrm{keV}$, quant à eux, interagissent principalement avec les atomes de bore en émettant dans des directions opposées une particule alpha et un noyau de lithium.

Les particules chargées (protons, alpha ou noyau de lithium) sont détectées dans la diode silicium et y déposent tout ou partie de leur énergie. L'analyse du spectre d'impulsions produites dans la diode permet l'évaluation de l'équivalent de dose et du débit d'équivalent de dose neutron en temps réel.

\section{Discrimination neutron-gamma}

La difficulté principale d'un tel détecteur réside dans la nécessité de discriminer les neutrons et les gamma en champ mixte. Cette difficulté liée à la physique du détecteur a conduit le service de dosimétrie de l'IPSN à étudier le comportement des charges électriques dans le silicium. Des méthodes permettant de séparer les impulsions dues aux neutrons de celles dues aux photons ont été développées. L'IPSN a retenu la méthode la mieux adaptée aux besoins et a engagé, courant 1998, le transfert de technologie vers la société Saphymo.

\section{Consommation d'énergie}

En vue de l'industrialisation du dosimètre, les contraintes d'encombrement et d'autonomie énergétique ont été prises en compte. Pour maintenir la consommation d'énergie au niveau le plus bas, la société Saphymo a développé en collaboration avec le $\mathrm{SEI} *$ une électronique intégrée de type «ASIC ». Ce choix technologique permet de réduire le bruit de fond électronique et d'adopter des solutions technologiques novatrices en matière de détecteur. Une simulation de l'interaction des photons dans le silicium, à l'aide d'un code de calcul Monte-Carlo, a permis de déterminer la géométrie idéale de la diode, en l'occurrence un détecteur silicium dont l'épaisseur est suffisamment faible pour que les photons ne puissent pas déposer une énergie supérieure à une centaine de keV. Cette valeur correspond au seuil en dessous duquel les particules chargées ne sont plus prises en compte lors de l'évaluation de l'équivalent de dose. En collaboration avec la société Canberra, une diode silicium épitaxié multipistes a été conçue. La séparation des photons et des neutrons est effectuée par l'application d'un simple seuil en amplitude sur les impulsions. Ce nouveau détecteur a été validé en l'associant à une électronique discrète dérivée d'applications spatiales. Il a confirmé que les valeurs de réjection souhaitées étaient atteintes.

\footnotetext{
* Service d'écctronique et d'informatique du département d'astrophysique, de physique des particules, de physique nucléaire el d'instrumentation associée (DAPNIA/Saclay).
} 
On peut apprécier sur l'éclaté du dosimètre (Fig. 3) le faible encombrement de l'électronique d'acquisition et du capteur devant le volume de l'accumulateur. L'ensemble des calculs est réalisé par un micro-contrôleur. Une mémoire de grande capacité est couplée au micro-contrôleur, permettant ainsi de conserver les données acquises sur plusieurs cycles de mesures.

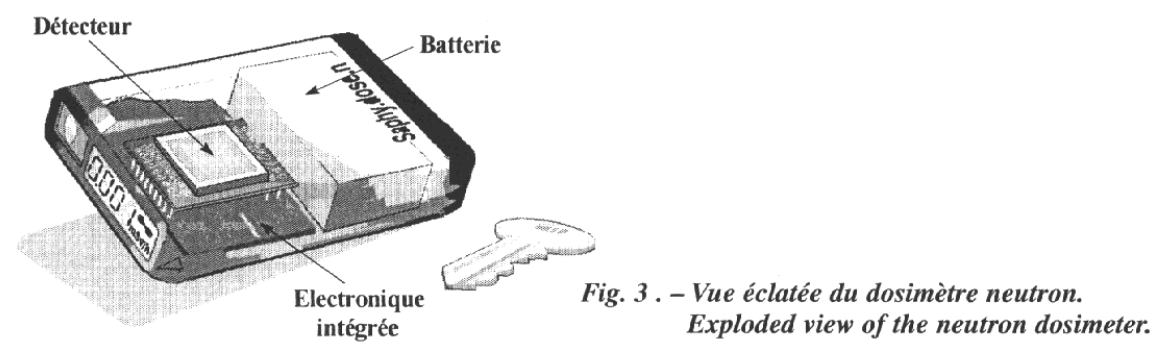

\section{Étalonnage}

Une série d'essais a été réalisée en neutrons mono-énergétiques (CEA/DAM/ DPTA à Bruyères-le-Châtel) et dans des spectres réalistes (installation Canel, IPSN/SDOS à Cadarache). Les résultats expérimentaux obtenus sont présentés sur la figure 4.

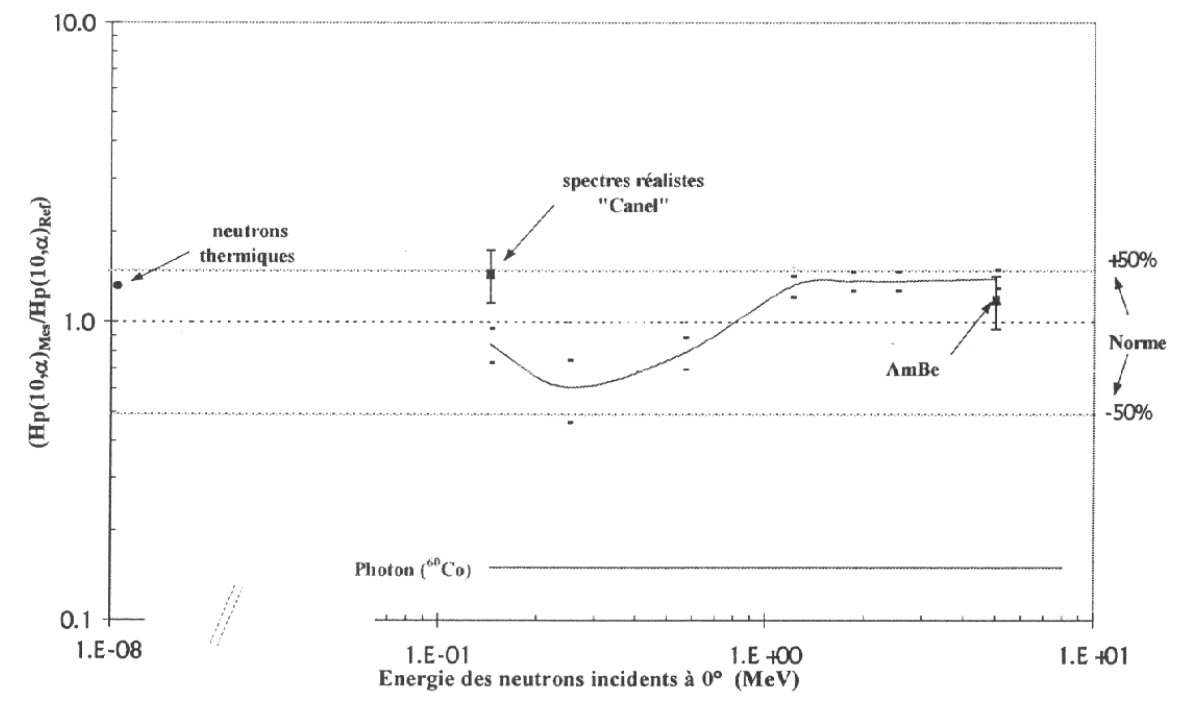

Fig. 4. - Réponse du prototype en termes d'équivalent de dose.

Dosimeter response in terms of dose equivalent. 


\section{Caractéristiques techniques du dosimètre industriel Saphydose-n}

La société Saphymo commercialisera fin 2000 le premier dosimètre individuel électronique pour les neutrons, le Saphydose-n, répondant à la norme CEI 1323 et au cahier des charges défini par le groupe de travail « dosimétrie neutrons ». Ce dosimètre, d'un encombrement réduit, permettra d'évaluer l'équivalent de dose $H p(10)$ dans des champs mixtes neutron-gamma. Il pourra être utilisé dans toutes les installations du cycle électronucléaire, sans connaissance préalable de l'énergie moyenne du spectre neutron et du rapport neutron/gamma. Les caractéristiques techniques reportées dans le tableau ci-dessous seront celles du dosimètre commercialisé fin d'année 2000 par la société Saphymo.

\section{Caractéristiques de mesures :}

\begin{tabular}{|c|c|}
\hline Gamne d'energie & Thermiques à $15 \mathrm{MeV}$ \\
\hline Étendue de mesure du débit d'équivalent de dose & $10 \mu \mathrm{Sv} \mathrm{h}^{-1}$ à $0,1 \mathrm{~Sv} \mathrm{~h}^{-1}$ \\
\hline Etendue de mesure d'écuivalent de dose & $1 \mu \mathrm{Sv}$ à $1 \mathrm{~Sv}$ \\
\hline Ecart maximal sur la réponse en fonction de l'énergie & $\pm 50 \%$ sur toute la gamme d'énergie \\
\hline Erreur maximale sur la mesture de débit d'équivalent de dose & $\pm 30 \%$ de $10 \mu \mathrm{Sv} \mathrm{h}{ }^{-1}$ a $100 \mu \mathrm{Sv} \mathrm{h}^{-1}$ \\
\hline & $\pm 20 \%$ de $100 \mu S v h^{-1} a 0,1 S v h^{-1}$ \\
\hline 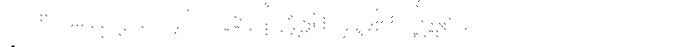 & $\pm 15 \%$ de $0,1 \mathrm{~Sv}^{-1}$ à $1 \mathrm{~Sv} \mathrm{~h}^{+1}$ \\
\hline Écart maximal sur la réponse angulaire (avec source $\mathrm{AmBe}$ ) & $\pm 30 \%$ entre $+75^{\circ}$ et $-75^{\circ}$ \\
\hline Sensibifte aux photons en $\mathrm{Hp}(10)$ & $<5 \%$ \\
\hline
\end{tabular}

\section{Caractéristiques générales :}

\begin{tabular}{|ll|}
\hline Dimensions & $130 \mathrm{~mm} ; 70 \mathrm{~mm} ; 25 \mathrm{~mm}$ \\
Masse & $<200 \mathrm{~g}$ \\
Alímentation & accumulateur lithium-ion \\
Autonomie & $>24 \mathrm{~h}$ \\
Affichage & equîlvalent de dose et débit d'équivalent de dose sur 4 caractères \\
Alarmes & visuelles par diode électroluminescente \\
& sonores de niveau $>80 \mathrm{dBA}$ à $30 \mathrm{~cm}$ \\
Lecture et gestion & compatible avec les blocs chargeurs et les lecteurs Saphymo existants \\
\hline
\end{tabular}

À ces caractéristiques, s'ajoutent certaines fonctions : information sur les composantes du spectre neutronique (thermique, intermédiaire, rapide) et distribution des énergies déposées dans le détecteur. L'ensemble de ces informations servira lors d'analyses « post-intervention » effectuées, par les services de radioprotection. Les dosimètres Saphydose-n existeront, soit en version compatible avec les bornes existantes des dosimètres gamma pour la lecture des informations et pour la charge des accumulateurs, soit en version autonome. Une étude d'intégration du dosimètre gamma dans le boîtier du dosimètre neutron est en cours. 


\section{RÉFÉRENCES}

Bordy J.M., Lahaye T., Landre F., Hoflack C., Lequin S., Barthe J. (1997) Single diode detector for individual neutron dosimetry using pulse shape analysis. $8^{\mathrm{e}}$ symposium sur la dosimétrie des neutrons, 1995. Paris, Radiat. Prot. Dosim. 70, 73-78.

Chartier J.L., Janski B., Kluge H., Schraube H., Wiegel B.(1997) Recent developments in the specification and achievement of realistic neutron calibration fields. $8^{\text {e }}$ symposiun sur la dosimétrie des neutrons, 1995, Paris, Radiat. Prot. Dosim. 70, 305-312.

Fernadez F., Lugera E., Domingo C., Baixeras C. (1997) Separation of the neutron s.gnal from gamma component in (n-g) fields using differential pulse analysis techniques with a double silicon diode. $8^{e}$ symposium sur la dosimćtrie des neutrons, 1995, Paris, Radiat. Prot. Dosim. 70, 87-92.

ICRP Publication 60 (1991) Ann. ICRP 21 (1-3).

Norme ISO 8529 : Rayonnements neutroniques de référence destinés à l'étalonnage des instruments de mesure des neutrons utilisés en radioprotection et à la détermination de leur réponse en fonction de l'énergie des neutrons.

Norme CEI 1323 : Instrumentation pour la radioprotection-Rayonnements ne utroniques-Moniteur individuel à lecture directe d'équivalent de dose et/ou de débit d'équivalent de dose.

Rannou A., Clech A., Devita A., Dollo R., Pescayre G. (1997) Evaluation of individual neutron dosimetry by a working group in the French nuclear industry, Radiat. Prot. Dosim. 70, 181-186.

Rannou A., Barthe J., Aubert B., Brégeon B., Champlong J., Colson P., Espagnan M., Herbaut Y., Thevenin J.C., Valero M. (1998) Etat de l'art des techniques de dosimétrie individuelle ct analyse des besoins, Radioprotection 33, 405-433. 